\title{
Erratum to: Copy number variation in the human Y chromosome in the $\mathrm{UK}$ population
}

\author{
Wei Wei ${ }^{1}$ ' Tomas W. Fitzgerald ${ }^{1} \cdot$ Qasim Ayub $^{1} \cdot$ Andrea Massaia $^{1} \cdot$ Blair H. Smith $^{2}$ • \\ Anna F. Dominiczak ${ }^{3} \cdot$ Andrew D. Morris $^{4} \cdot$ David J. Porteous $^{5}$ • \\ Matthew E. Hurles ${ }^{1} \cdot$ Chris Tyler-Smith $^{1} \cdot$ Yali Xue $^{1}$
}

Published online: 19 May 2015

(C) Springer-Verlag Berlin Heidelberg 2015

\section{Erratum to: Hum Genet \\ DOI 10.1007/s00439-015-1562-5}

The second author's initial was missing, and the fifth to eighth authors' initials were incorrect. The correct names are Tomas W. Fitzgerald, Blair H. Smith, Anna F. Dominiczak, Andrew D. Morris, and David J. Porteous.

The complete author list is given below:

Wei Wei, Tomas W. Fitzgerald, Qasim Ayub, Andrea Massaia, Blair H. Smith, Anna F. Dominiczak, Andrew D. Morris, David J. Porteous, Matthew E. Hurles, Chris Tyler-Smith, Yali Xue

The online version of the original article can be found under doi:10.1007/s00439-015-1562-5.

Yali Xue

ylx@sanger.ac.uk

1 The Wellcome Trust Sanger Institute, Wellcome Trust Genome Campus, Hinxton, Cambridgeshire CB10 1SA, UK

2 School of Medicine, Ninewells Hospital and Medical School, Dundee University, Mackenzie Building, Kirsty Semple Way, Dundee DD2 4RB, UK

3 College of Medical, Veterinary and Life Sciences, University of Glasgow, Glasgow G12 8QQ, UK

4 School of Molecular, Genetic and Population Health Sciences, University of Edinburgh Medical School, Teviot Place, Edinburgh EH8 9AG, UK

5 Institute of Genetics and Molecular Medicine, Western General Hospital, University of Edinburgh, Crewe Road South, Edinburgh EH4 2XU, UK 